\title{
Porous Nitrogen-Doped Carbon Nanoribbons for High-Performance Gas Adsorbents and Lithium Ion Batteries
}

\author{
Quan-Sheng Yang, ${ }^{\text {a,b }}$ Zhu-Yin Sui, ${ }^{b}$ Yu-Wen Liu, ${ }^{, a}$ and Bao-Hang Han, ${ }^{*}$ \\ ${ }^{a}$ Department of Environment and Chemical Engineering, Yanshan \\ University, Qinhuangdao 066004, China \\ ${ }^{b}$ CAS Key Laboratory of Nanosystem and Hierarchical Fabrication, CAS \\ Center for Excellence in Nanoscience, National Center for Nanoscience and \\ Technology, Beijing 100190, China
}

Tel: +8610 8254 5576. E-mail: hanbh@,nanoctr.cn.

Tel: +86 3358061 569. Email: liuyuwen@ysu.edu.cn. 


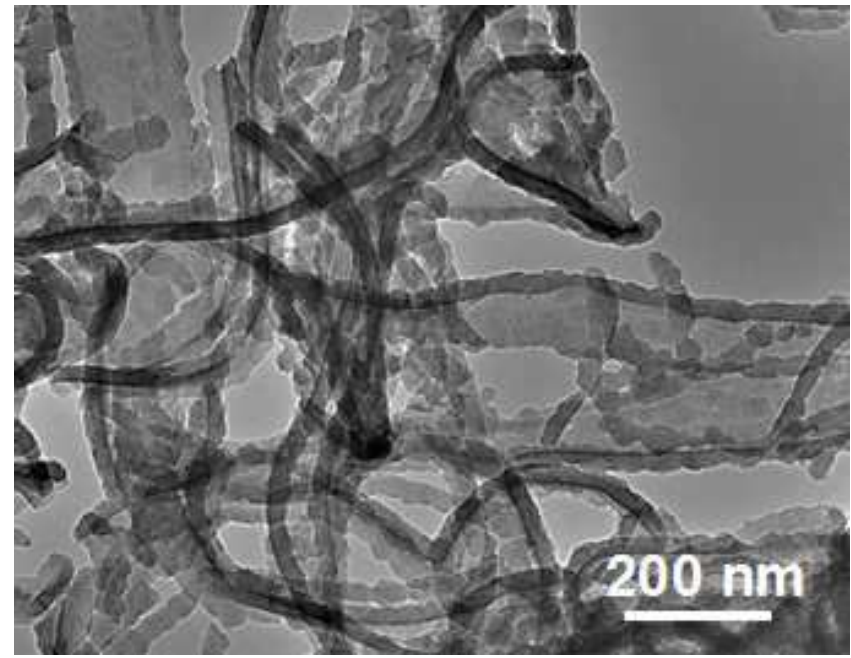

Figure S1. TEM image of PPNs. 


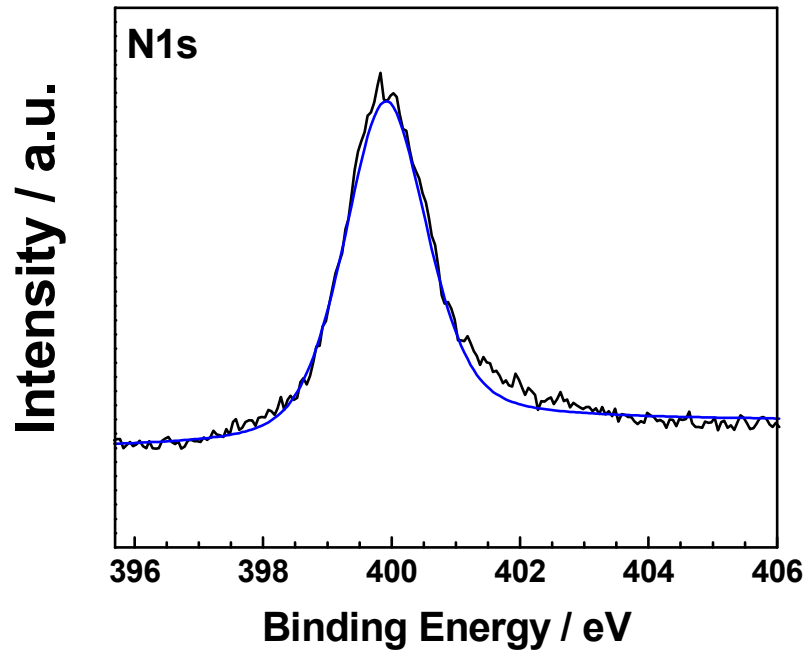

Figure S2. XPS N1s spectrum of PPNs. 


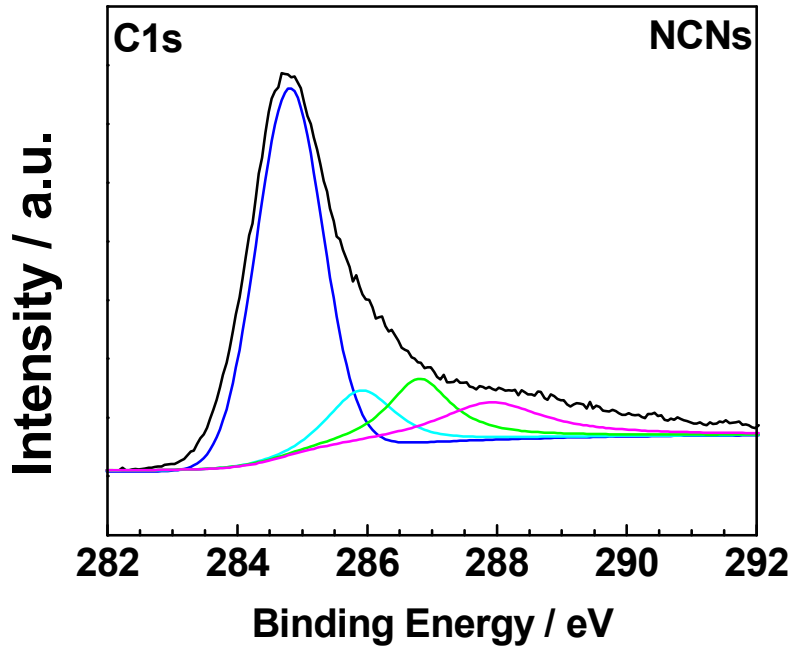

Figure S3. XPS C1s spectrum of NCNs. 


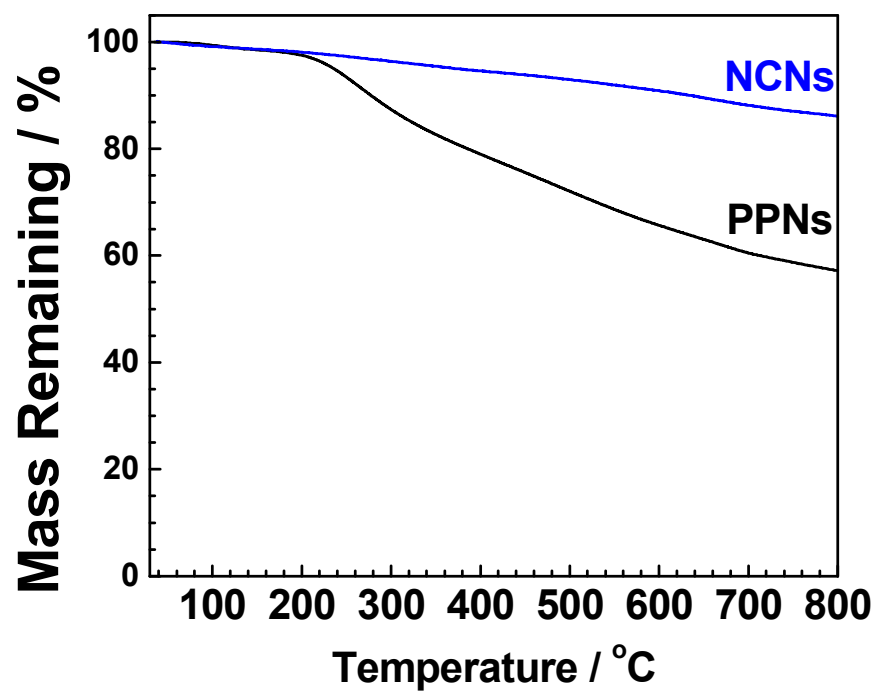

Figure S4. Thermal gravimetric analysis curves of NCNs and PPNs under nitrogen atmosphere. 


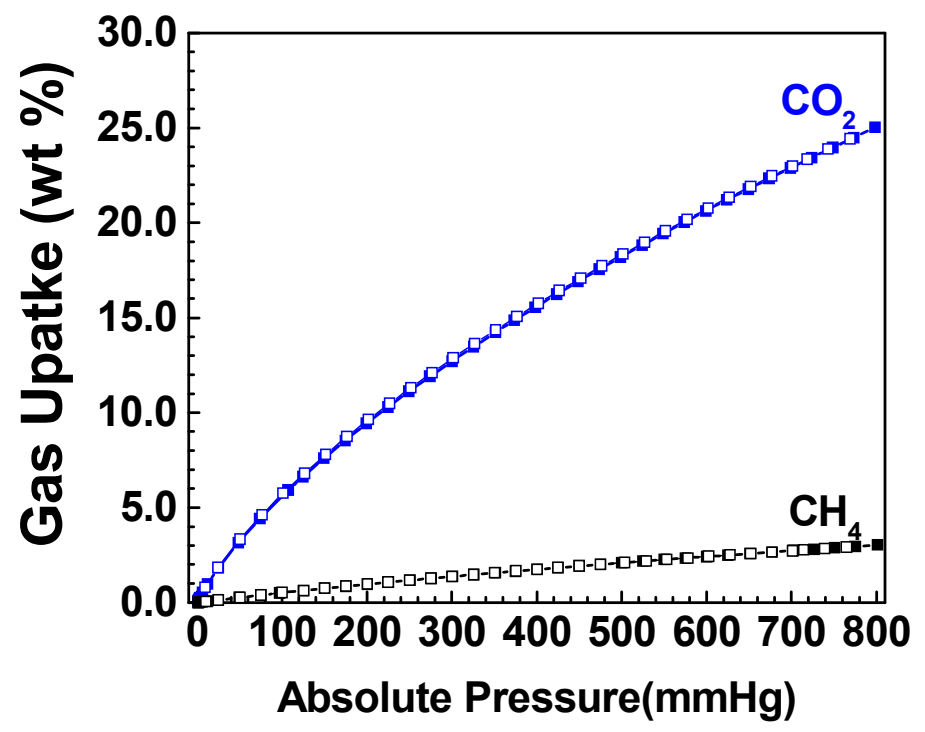

Figure S5. Carbon dioxide and methane adsorption-desorption isotherms of NCNs at $273 \mathrm{~K}$ (solid symbols for adsorption and empty symbols for desorption). 


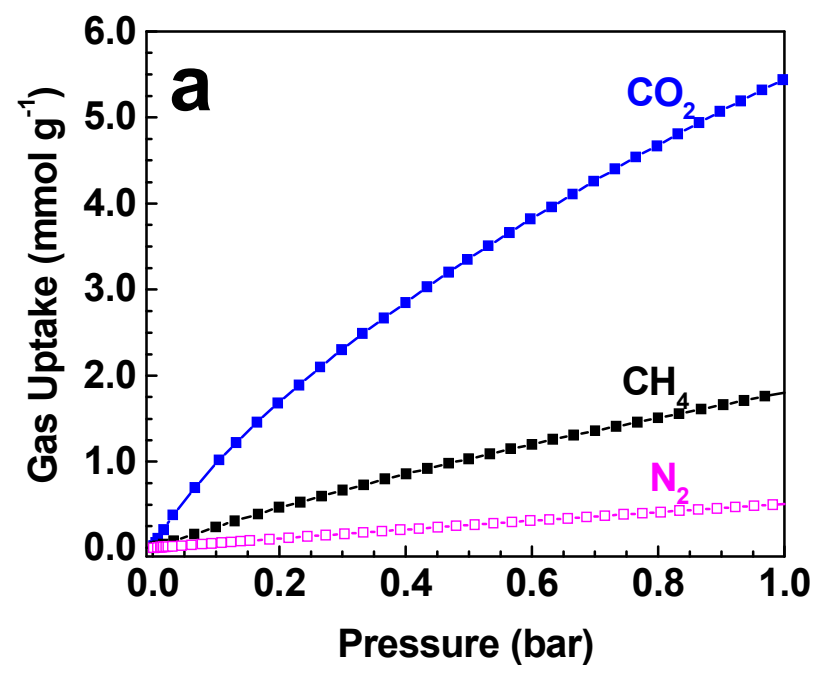

Figure S6. Carbon dioxide, methane, and nitrogen adsorption isotherms of NCNs at 273 K. 


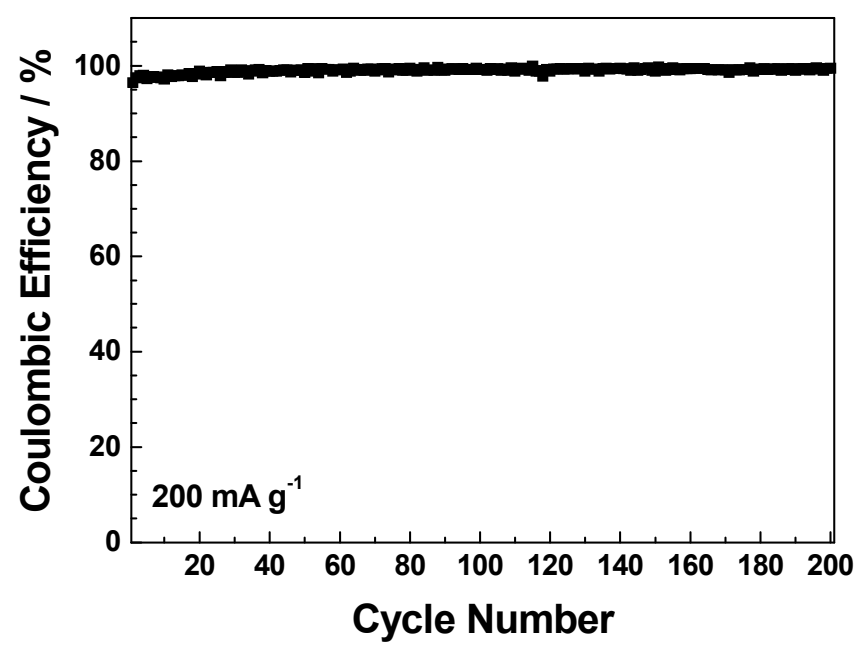

Figure S7. The Coulombic efficiency of NCNs at a current density of $200 \mathrm{~mA} \mathrm{~g}^{-1}$. 